\title{
Pandemic Fatigue: A Concept Analysis
}

\section{Elvira Teng-Costa ${ }^{1}$, Catherine M. Macatangay ${ }^{1}$, Neil N. Balubar ${ }^{1}$, Roison Andro Narvaez ${ }^{1}$}

\author{
${ }^{1}$ Saint Paul University, Philippines
}

Corresponding Author: Roison Andro Narvaez

DOI: https://doi.org/10.52403/ijhsr.20220312

\begin{abstract}
Aim: This concept analysis aims to impart knowledge and recognize the pandemic fatigue of healthcare professional, especially nurses. The concept aims to identify the causes of pandemic fatigue to nurses, especially during the COVID-19 crisis. It also identifies the effect of pandemic fatigue on their productivity, their mental and physical aspect. It also analyzed the definitions of the concept via various case studies and researches like giving examples of the model, related, contrary and borderline cases.

Background: Pandemic fatigue is a foreseeable result of an extended health crisis. The gravity of its impact on people's lives and the seriousness of the disease signaled different measures to combat the infection. The pandemic fatigue can be seen from the records of various countries' growing numbers of individuals who do not adequately conform to given measures, are not aware of the COVID-19 disease, and their exhaustion of the changing safety protocols.

Design: Avant and Walker (2019) method of concept analysis

Result: The study has uncovered seven significant contributing attributes of pandemic fatigue, especially healthcare workers, such as exposure to infection, restriction, mortality, relationships with coworkers, employers and public, PPE/supplies, and nursing shortage mental health. Pandemic fatigue among nurses has been rampant and has affected the healthcare workforce's daily routine. Prolonged crisis has set the government in a pinch and different hospitals in both private and public.

Conclusion: Nurses are prone to developing pandemic fatigue due to their nature of work and the additional burden COVID-19 has brought. Pandemic fatigue can be prevented initially by studying fatigue's underlying cause and formulating strategies to help nurses overcome pandemic fatigue. It can be prevented by following the established guidelines and support from the family and the nurses' institution. Furthermore, the nurse can develop their behavior on what is suitable for them to control fatigue.
\end{abstract}

Keywords: pandemic fatigue, COVID-19, nursing, concept analysis, Avant and Walker

\section{INTRODUCTION}

The spread of COVID-19 worldwide impacted everyone and, more specifically, those directly infected with the virus. It is of great concern among nurses as it is spreading rapidly amid the Coronavirus Disease 2019 widespread, the front line nurses' experience immense health struggles. Nurses are one of the professions that are primarily responsible for taking care of those infected with COVID-19, and they are more prone to contracting the disease, thus creating danger to their health, which impacts their life in all aspects. One of its effects is pandemic fatigue. It is an anticipated and typical reaction to a troublesome circumstance like this pandemic. It is a health concern by many people during COVID-19.

Pandemic fatigue is characterized as both physical and mental exhaustion resulting from disturbance from the usual 
activities of daily living of a person and the different guidelines set to limit the spread of disease. [1] A study was done by the International Council of Nurses [ICN] (2021) illustrated that a considerable percentage of National Nursing Associations (NNA's) gave back records of mental health problems coming from nurses that work in line with COVID-19. [2] Nurses detailed feeling detached from their loved ones and afraid of spreading the coronavirus to their family members. Some NNAs have gotten reports of extended nurses' duty hours and facing mental distress because of a lack of protective gear. Differing studies stated that the health crisis worsens the stress and tiredness faced by the nurses. At the start of the pandemic in China, most nurses detailed fatigue as showed up in emotional exhaustion and depersonalization. [3] The occurrence is complicated and comes from a variety of issues, namely the amount of work they render, reliance and deaths of patients, stress from the job itself, lack of PPE's, worries that they may spread the disease to the family, unfair treatment of the society to nurses, lack of reliable data regarding the condition, and lastly they don't have enough support mentally and socially (ICN, 2021).

The effect of COVID-19 could expand the figure of nurses coming to exhaustion and quitting the job; the forecast would be that there would be a lack of nurses at around 14 million in the coming years. The majority of the healthcare population all over the world are nurses. Policymakers have to increase the negative impact of the crisis on the maintenance of people working on healthcare and the probable danger to worldwide well-being. International Council of Nurses (2021) asks the authority to require immediate measures to guarantee the physical and mental wellbeing of all healthcare workforce to develop resilience and create a policy to respond to the worldwide nursing deficiency [2].

In short, the spread of COVID-19 worldwide had a significant influence on the people, especially the nurses who have direct contact with the COVID-19 patients. It can cause pandemic fatigue to many people and healthcare workers. It is a foreseeable event in a particular situation like this pandemic and is a health concern by many individuals. Pandemic fatigue is defined as the physical and mental tiredness because of disruption from the typical activities of an individual and a variety of protocols implied to limit the spread of the disease. Thus, its impact could be the depletion of nurses and the possibility of changing their careers. [4]

Thus, this concept analysis aims to impart knowledge and recognize the pandemic fatigue of healthcare professional, especially nurses. The concept aims to identify the causes of pandemic fatigue to nurses, especially during the COVID-19 crisis.

\section{Definitions and Uses of Concept}

According to [5], the pandemic is characterized as (of a disease) present in nearly all of a group of people, animals, or plants. At the same time, fatigue means extreme tiredness [6]. The concept of pandemic fatigue has been much more emphasized after several months of struggling to beat COVID-19. The definition of pandemic fatigue from different standpoints or perspectives has been collected. It came from public health experts, WHO, a psychiatrist, a few individuals, and nurses.

The Public health experts call this phenomenon "pandemic fatigue" because they witnessed a consistent decline in compliance with COVID-19 mitigation behaviors over time. [7]. For them, the concept of pandemic fatigue is taxing because it involves the government and the people. In this present situation, it can be said that it is not about the tiredness or lack of resources, but the people's attitude regarding the guidelines implied to follow. The authorities would recommend measures like wearing of face mask during a pandemic, which is not a routine before the pandemic. Still, as people learn to 
understand the importance of such measures, they will add them to their daily practice.

Additionally, pandemic fatigue has been defined as an anticipated and expected reaction to a delayed, complex situation that influences the population, according to the [8]. The seriousness of the COVID-19 has enabled the usage of different measures that have affected society's everyday routine. During the health crisis, for instance, the COVID-19 pandemic, WHO played a vital role in preventing and managing the present worldwide disease. The intricate nature of COVID-19 requires various plans that people must follow continually to prevent the spread of infection. It includes many concerns from public health, people, culture, and the resources available in a particular place. By knowing the factors as to why pandemic fatigue occurs, they may formulate strategies that would help the people and the government maintain the required behaviors during a pandemic.

Moreover, Dr. Paul Nestadt referred to the exhaustion one may feel following months of investing additional time and strength in the current way of life and all the battles it's brought on [9]. For Dr. Nestadt, he says that the pandemic doesn't have an exact time for when it will end. The people had experienced and reported significant life-changing situations that made them tired. Moreover, It includes physical and mental exhaustion that results in negative feelings [9]. Understanding the underlying factors of suspicious behavior of people, he then gave some tips to cope with stressors one may be facing.

From a professor's idea, it can be defined as a community issue since it brings out people not to follow the guidelines set by the authority, situating themselves and the people around them at hazard. [10]. It is said that for the past years, the pandemic has changed the lives of people differently. There are available vaccines, but it seems that the disease is still unending with new variants being discovered. Overall, people are very tired of the situation, but there are some measures that people can take to help cope with the pandemic cautiously.

\subsection{COVID-19}

The remarkable increase in COVID19 cases worldwide has influenced individuals of all countries. The seriousness of the disease has significantly expanded and challenged the obligations of healthcare workers to ensure the well-being of the people; thus, its spread arises immediate response for the detection, diagnosis, treatment, management, and avoidance of infection [11]. Working in this crisis is difficult for nurses as they have the ultimate obligation to care. They are more prone to contracting the disease and infecting their families.

\subsection{Psychological Stress}

Healthcare workers, especially nurses, these people who experienced psychological stress and distress, according to [12], who are at risk of developing the disease. The health crisis has ceased services dedicated to mental health in almost all countries globally. Those nurses directly working in COVID - 19 faced challenges like heavy workload, tiredness, exposure to infection, sadness related to the death of a handled patient, and struggle communicating with family members of the patient added stress to nurses [13]. This situation can reduce nurses' motivation to work during this pandemic. Demotivated nurses tend to resign, and others choose to change the path in their careers. Nurses must step up or challenge themselves to cope with the added job or new work environment. Training must be done regarding handling infected patients to protect healthcare workers. Additionally, nurses' psychological health should be taken into consideration.

\subsection{Negative Experience of an Individual}

During this pandemic, it is typical to experience bad dreams, outrage, annoyance, and recall of events brought by COVID-19like deaths and being infected. The 
COVID-19 pandemic can be compared to a post-traumatic stress disorder that an individual sees a possibility of war, witnessed a crime, or grievous acts [14].

The pandemic had caused isolation and fear that could affect an individual's mental health. Some opt not to go out and stay at home to ensure that they will not acquire the disease. They sometimes give up on pursuing their career because of anxiety. People procrastinate on their plans because the situation is not yet normal, and it is difficult to work during this health crisis.

\subsection{Depression}

Depression is among the usual identified mental disorders, and healthcare workers have a greater chance of having it. [15] At this time of the pandemic, it is frequent to hear that healthcare workers who work with COVID-19 patients often feel depressed. Nurses are afflicted with this condition for many reasons in the work environment. The function of nurses is not limited to caring; they also coordinate multidisciplinary care over different services in the management of the disease to guarantee the control of the pandemic [16]. During the health crisis, nurses have worked beneath constraints, adversely affecting their health.

\subsection{Mortality and morbidity}

Previous reports show that the spread of infection likely happens with close contact with those infected with the COVID-19. [17] COVID-19 disease and mortality among health workers are common worldwide. [18] Healthcare workers assigned directly with COVID-19 patients are riskier of acquiring the disease. An example would be handling patients that require aerosol-generating procedures like suctioning. On the other hand, personnel working in different areas should not be underestimated as there is also a high chance of contracting the infection. Early identification and management of the disease would help prevent the spread and protect the patient and the facility.

\subsection{Personal Protective Equipments (PPE)}

There's a limited supply of PPE, and from time to time, there is a confusing policy regarding its uses and recommendations. [19] Consequently, it brings anxiety among nurses and other healthcare personnel. Incorrect utilization of PPE can cause contamination and burden the institution they work with. PPE's should be distributed appropriately among those high-risk areas, and continuous study must be made for its proper usage. The nurses play an essential part in the use of PPEs by limiting staff use, education, and training. In the future, nurses can help the authorities identify the purpose and proper use of PPE's.

\subsection{Restrictions}

The imposition of measures to limit the spread of COVID-19 continues as there is an increase in the number of people infected [20]. Community locks down were implemented worldwide with specific ordinances per country. It is believed that it helps to limit the spread of the infection. Travel restrictions, the closing of establishment, and curfew were done in each locality as needed. Furthermore, it impacted the lives of many, for instance, the economy, and many people lost their job due to the closure of their workplace.

\subsection{Relationship with coworkers, employers, and the public.}

The pandemic had changed the working conditions in numerous ways. The working environment can play a significant part in the well-being of an individual. It includes the relationship with the employers and the colleague. Furthermore, it contains unstable jobs, future unpredictability, and prolonged isolation. [21] The employers can help by ensuring a safe working environment, implementing safety measures as per guidelines, and educating the staff regarding training and updated lectures on COVID-19. 


\subsection{Nursing shortage}

There's a worldwide deficiency of nurses, and there is an increased demand for nurses to retire. [22] The migration of nurses is one cause of the shortage. Nurses from third-world countries seek a higher income than their home country. Another reason is inadequate quality education and training for the incoming students who wish to pursue nursing. Moreover, the aging population of nurses contributes to the shortage.

\subsection{Social isolation}

Lockdowns have been imposed globally since the pandemic, resulting in alteration in usual routine and reduced opportunity to socialize. [23] Since nurses are considered front-line workers in the battle to stop the spread of the disease, they are also at risk of contracting it and spreading the disease to the family and community. Consequently, they opt to keep isolated from their loved ones. The lack of interaction with family members and other people could be a reason for nurses' stress resulting in added health problems.

\subsection{Risk level}

The nurses have an increased risk of exposure because they give personal care to COVID 19 patients. For instance, they extract blood samples or specimens to detect the virus or do any aerosol-generating intervention, together with not adhering correctly to the set guidelines [24].

\subsection{Mental Health}

The impacts of pandemics on mental health are probably significant and would stay for a long time. [25] Healthcare workers responsible for the COVID 19 patients have a greater risk of having mental health problems like stress, exhaustion, and post-traumatic stress disorders. [26] The institution where nurses work must assess the factors that lead to nurses' mental health problems to protect and maintain their health. Despite the guidelines established in disease management, the personnel working in combating the disease are not used to the psychological effects. The front liners are directly affected and have an increased risk compared with other healthcare workers.

\subsection{Burnout}

The issue of burnout among healthcare workers is present even before the pandemic and even rises in number during the ongoing COVID 19 infection. [27] The possible causes of this are the extra or extended work of the nurses, unfamiliarity with the given duties, and most especially if they cannot get the support from the institution, they are working. Burnout could result in depression, stress, and PTSD. [28] Burnout among healthcare workers should be emphasized, giving the appropriate interventions to cope with the challenging situation.

\section{METHODS}

Walker \& Avant's (2019) step model of concept analysis was used. The concept analysis method of Walker and Avant (2019) is commonly used for nursing research and has eight step. [29] First is selecting the concept, second is determining the aims or the purpose of the concept, then identifying all the uses of the concepts that can be discovered, then choosing the defining attributes, followed by identifying the case model such as borderline, related, contrary, and lastly identifying antecedents and consequences and lastly defining the empirical referents. [29]

\section{Data Collection}

Data collection comprises different parts of the concept analysis. Databases searched were the World Health Organization (WHO) and the National Library of Medicine (Research fatigue in COVID-19). Journals databases such as Google Scholar, Pubmed, EBSCO Host, Sage Journals, and Cumulative Index to Nursing and Allied Health (CINAHL) were used in searching literature review. It includes the defining attributes of the 
concept, which is considered the center of the idea and thus serves as a basis for the formulation of model cases, related cases, borderline cases, and contrary cases, including their respective analysis. It covers the antecedents, empirical referents, and consequences to describe pandemic fatigue.

\section{Defining Attributes}

Pandemic fatigue in nursing was defined with different articles and studies available. Nevertheless, it was said that there is no clear definition of pandemic fatigue in nursing. The purposes came from various individuals who experienced fatigue during the pandemic. There are also reports like surveys from nurses worldwide about their perception of pandemic fatigue. Regardless of this, the concept of pandemic fatigue can be clearly described by looking at its defining attributes. The study has uncovered seven significant contributing factors in pandemic fatigue, especially healthcare workers, such as exposure to infection, restriction, mortality, relationships with coworkers, employers and public, PPE/supplies, and nursing shortage mental health.

\subsection{Exposure to Infection}

Exposure to infection means the fear of getting sick and spreading the disease to other people. Specifically, it includes the worry of transmitting the infection to their loved ones or patients.

\subsection{Restrictions}

Restrictions are also considered as one of the factors causing not only to our health workers but as well to other people, closure of the establishment such as groceries; some markets, restaurants caused insecurities and fear of the unknown as the majority of the people got their basic needs in the indicated places, transportation, mainly those going to the provinces also are canceled due to stringent securities along borders.

\subsection{Mortality}

This topic speaks about managing an individual's disease, sickness, and passing, more often than not, patients, friends, or cherished ones. It incorporates seeing the deterioration of patients, seeing their deaths, and caring for ventilated or enormously sick patients. Moreover, it includes pressure to keep ill patients separated from their family and their adored ones.

\subsection{The Relationship with Coworkers and Employers, and public}

It describes the environment where a person performs their job. It includes their relationship with their workmates, the workloads, the unfamiliarity of handling the disease, and the lack of training or support from the institution they worked with. According to [30], the illustration would be like creating remote working rules, reducing working hours and salaries, temporary suspension of business activities, permanent termination of contracts, and suspension of businesses.

\subsection{PPE/Supplies}

This topic describes stressors related to PPE/supplies. This incorporates not having sufficient PPE, ventilators, and testing supplies. In addition, it includes having to re-wear PPE, conflicting PPE rules, and physical inconvenience related to wearing PPE. An example of a nurse reaction would create a question and concern about whether our health system will be able to fully protect our health care workers. [31]

\subsection{Nursing Shortage}

Nurses are vital to the healthcare society, and they have the most significant proportion among the healthcare workers. However, the profession also experiences a shortage of workforce due to various reasons, including lack of quality education, clinical instructor, unfair treatment, and more opportunities outside the profession [32]. The pandemic results in an additional burden to the nurses, and the nurse-patient 
ratio can see this. At times, the work given is too much to be handled by the healthcare worker that causing them fatigue.

\subsection{Mental Health}

In times of health crisis, an individual's health is greatly affected. An individual's reaction to the situation contributes to their psychological health, and some of the responses are distress and dysfunctional behaviors. [33] In addition, their response is connected to how they will adhere, manage and cope with the threat of the disease. Nurses who did not adhere to the set guidelines have an increased level of depression, tension, and concern for their health. [34]

\section{CASE STUDIES}

\subsection{Model Case and Analysis}

Walker \& Avant's (2019) Model case exemplifies the concept that exhibits or shows all the defining attributes. It is a pure case of the concept or a pure exemplar. [29]

Cath is almost five years in the field of nursing, her perception and feeling about her work were always the same until COVID-19 came. Catherine says that she experienced physical and emotional exhaustion in handling COVID patients, and she has always had a fear of the possible consequences. She feared contracting the disease or carrying the virus and transmitting it to her family; moreover, she feels that the time she spent with her family and their bonding was lessened as she isolated herself. She also feels tired because of an increasing number of patients requiring complete PPE in giving the needed critical care; she feels great stress and the heavy burden of her increasing responsibilities. She continuously feels that there is a sense of defenselessness and recoups from these impacts.

\section{Analysis:}

The case model consists of all defining attributes of the concept because it discusses the experience of the individual healthcare workers, especially the nurses, about the struggles and challenges that COVID 19 entails for them. As a front-liner dealing with the COVID, she shows all the fundamental traits of fatigue, including a decrease in well-being, and experiences physical fatigue and weakness.

\subsection{Related Case}

Walker \& Avant (2019) said that related cases in concept analysis consist of the defining attributes of the concept being studied, but not all of it. [29]

"Mike is a 20-years old who was assigned at the district hospital. He decided to work in one of the busy wards, where they handle 20 patients per shift. He works in the facility and deals with different problems, such as straight night shifts with only one day's rest. It is difficult for him due to limitations of freedom, and sometimes he cannot do personal things as he needs to rest only on his off days. The meager salary and benefits also increase here burden, and sometimes he thinks it is much better to shift to another kind of work or work abroad".

\section{Analysis:}

Typically, a related case does not contain all the fundamental properties of pandemic fatigue. It is acceptable because the issue is related to the concept. In this case, out of the seven defining attributes cited under the concept of pandemic fatigue, two are shown here, like restriction and nursing shortage. The subject experiences some adverse effects brought by the pandemic in his work.

\subsection{Borderline Case and Analysis}

Borderline cases are examples or instances that contain most of the defining attributes of the concept being examined but not all of them. They may contain most or even all of the defining characteristics but differ substantially in one of them, such as the length of time or intensity of occurrence. [29] 
"Ms. Joyce stated that "Despite all the physical and mental exhaustion I'm encountering, I always continuously motivate myself to do all my best to deliver quality healthcare to all patients. I also make sure that I always communicate with my family to maintain our bonds. In times of challenges like the death of a patient or coworkers contracting the disease, I always seek advice and help from my friends, colleagues, and family for support and motivation. I always enhance and update my knowledge and skill by attending different seminars and workshops."

\section{Analysis:}

The scenario shows a borderline case because although the subject shows the symptoms of stress at work, she could fight and cope with her condition through different coping mechanisms and support. Borderline cases are examples or instances that contain most of the defining attributes of the concept being examined but not all of them. They may contain most or even all of the defining characteristics but differ substantially in one of them, such as length of time or intensity of occurrence. Out of seven identified attributes, it includes some three attributes like exposure to infection, nursing shortage, and relationships with coworkers

\subsection{Contrary Case}

Contrary cases are clear examples of "not the concept." [29]

"Jose has been working as a medical-surgical nurse for almost five years; in this time of the COVID-19 pandemic, the work is quite stressful and always loaded compared to before the pandemic. Jose makes it a point that he avoids those things that makes him stressed. He takes a regular dose of vitamins, hydrates himself well, and ensures enough rest and sleep. He is completely vaccinated against the COVID-19 vaccine. In times, that some of his colleagues can't go to work, and if he can relieve them, he then goes to work. Given the present situation, he still is energized and renders his duties and responsibilities as a nurse to give quality patient care. Jose has a passion for his job, and he is optimistic about the circumstances he faces by thinking of ways to manage his work so that he will not be fatigued".

\section{Analysis:}

This case was considered contrary because he displayed no essential attributes of pandemic fatigue. Being a nurse handling COVID 19 is highly stressful, but he uses positive ways to protect himself from pandemic fatigue.

\section{Antecedents}

Antecedents occur before the concept emerges that offer perspective on the social context in the central concept under analysis. [29] There are antecedents in the literature that were found related to pandemic fatigue which is COVID-19 pandemic, social isolation, risk level, and burnout.

\subsection{COVID-19}

The gravity and severity of the disease bring about fear and tension among nurses that could affect their health and job during this difficult time. [35] In addition, those assigned to handling COVID patients routinely witnessed the deteriorating and dying patients that could impact their wellbeing and cause them fatigue (Alharbi et al., 2020) and post-traumatic stress. [36] A study done by [37] showed that almost a quarter of nurses were seen to have anxiety. A review of studies describes that nurses experienced a high level of anxiety and depression compared to other healthcare workers. [38]

\subsection{Social isolation}

The health crisis has caused a rise in social isolation worldwide, but it dramatically affects those working in the hospitals or those who cater to COVID-19 patients. This is because of some of the institution's infection protocols, like putting 
a patient in one room without a companion and restricting the visiting privilege. [39] Social distancing is a well-known guideline to combat the disease and is adequate in minimizing the spread of COVID-19. [40] Aside from that, social distancing reduces the bed capacity of the hospitals but has some impacts like reducing employees, limiting the number of patients and the clinic hours, and mental health problems. [41]

\subsection{Risk level}

An analysis of data from the UK and the US illustrates that those who worked as front liners have more risk of contracting the disease than those individuals in the community and people whose job doesn't require direct exposure to the infection, for example, factory workers. Not only that, it is said that they are prone to having the disease and spreading it to their loved ones and the crowd. [31] According to some published reviews, factors contributing to the disease are lack of PPEs, handling positive patients, extended working time, and more loads of tasks. Other reasons for acquiring the condition are comorbidities and measures that don't conform with infection control prevention. [42]

\subsection{Burnout}

Burnout among nurses, particularly those working in the hospital, seems to bring a significant problem in rendering service to the patient. Globally, the workforce of nurses reports an increased level of burnout. [43] The results could lead to changing jobs or leaving their profession. The psychological well-being of nurses must be addressed to provide quality care to the patient.

\section{Consequences}

Consequences are events or incidents that occur due to the occurrence of the concepts, or in other words, the outcomes of the idea. [29]

Possible consequences of pandemic fatigue may include interrupted work routines and activities of an individual due to different measures done to help decrease the transmission of virus-like lockdown, curfews, and social distancing [1] and the resignation of staff nurses and other health care providers, feeling hopelessness with the increasing number of cases infected with COVID-19. This also leads other nurses and other healthcare workers to seek jobs not related to healthcare. Thus some opted to open and put up businesses that will make them feel more at ease, safe, and secure. The healthcare worker left in hospitals and quarantine facilities would also seek to increase salary for handling patients more than what is the recommended and of standard. On the bright side, this in a way awakens the government to give more importance to matters including health services, vaccines, and other health-related supplies in the coming years to be prepared for whatever pandemic may come in the future and invest more in the health sector for the betterment of the country of the people.

\section{Empirical Referents}

Empirical referents are classes or categories of actual phenomena that demonstrate the occurrence of the concept itself. [29]

Pandemic fatigue cannot be measured, but fatigue in general which [44] had developed, is a Multidimensional Fatigue Inventory where a 20 -item selfreport instrument designed to measure fatigue. This is divided into five dimensions: general fatigue, physical fatigue, mental fatigue, reduced motivation, and reduced activity. [45] also used this multidimensional fatigue scale with their article on the Pediatric Quality of Life Inventory (PedsQL), same as with Gordijn et al. (2011), a study that reports on feasibility, reliability, validity, and the norm-referenced of PedsQL Multidimensional Fatigue Scale of the Dutch. Multidimensional Fatigue Inventory (MFI) was also used to measure fatigue of different diseases such as pediatric cancer 
from the study made by [45] in 2002 and 2009 with diabetes Type $1[46,47]$.

Chalder Fatigue Scale measures the extent and severity of fatigue. [48] The selfadministered questionnaire was initially developed to measure the size of chronic fatigue, but [49] then revised it to measure the severity of 'tiredness' rather than just the chronic fatigue syndrome.

\section{DISCUSSION}

This pandemic on COVID-19 has affected the healthcare providers and the economy as well. A lot of businesses had been closed down. Education was interrupted, and families were separated and isolated. Pandemic fatigue is a worldwide phenomenon that affects mainly healthcare providers around the globe. The surge of patients exhausted the healthcare workers physically, mentally, emotionally, and socially. The burden did not end there.

Continuous discrimination of healthcare workers is continuously being experienced. Hospital and other facility staff are condemned despite doing the best they can be, going for the extra miles just to serve their fellow countrymen to get better passionately. The increased demand for oxygen and decreased supply, reduced availability of rooms, and short staffing; the impact of these scenarios and the consequences of the current condition gravely affected the people in the health sector in providing quality nursing care, which made them feel helpless in this situation. Uncooperative, close-minded, and selfish people further increased the frustrations and burden of healthcare providers. Pandemic fatigue does affect the person not only physically but also emotionally and mentally.

\section{Implication for Nursing}

The pandemic fatigue concept will help identify factors that significantly affect the nursing community and other healthcare providers and ways to prevent it. Further research and study may help the people be prepared for another pandemic that is yet to come.

COVID-19 Pandemic has been around the globe for a year in a half. In delivering care for patients infected with the coronavirus, nurses must learn to find ways to alleviate fatigue. Nurse managers can do programs on how to help the staff by coordinating with psychologists for counseling to help preserve nurses' mental health, which can help nurses function with conscience and presence of mind, thus preventing medical errors. Encouraging nurses to comply with vaccination will also help ease their worries about hospitalacquired infection and prevent nursing from getting seriously ill while rendering care to COVID-19 patients.

Nursing educators can prepare students taking up nursing on how to handle pandemic scenarios in the future. Diligence, vigilance, and professional ethics must be instilled and molded in the being of student nurses as early as possible. Nursing is not only a profession but a calling and a passion.

\section{CONCLUSION}

Different articles, journals, and studies have identified factors contributing to fatigue, particularly in this pandemic. The attributes identified in this analysis are exposure to infection, restriction, mortality, relationships with coworkers, employers, public, PPE/supplies, nursing shortage, mental health. The antecedents of pandemic fatigue are COVID-19, social isolation, risk level, burnout experiences of the healthcare workers.

Fatigue, in general, is more of a subjective term and varies from one person to another. Given their circumstance in which they need to stay at the hospital and be isolated from their loved ones, they can further increase their suffering and longing. Nurses tend to resign, go abroad for a better salary, and in some cases leave entirely behind nursing and change their careers altogether. Without giving proper attention and solution to the recent events and 
concerns that the medical world is experiencing, the health sector and the country itself will be in turmoil.

\section{Acknowledgement}

The authors acknowledge the support and guidance of the Graduate School of St. Paul University Philippines

\section{Conflict of Interest}

The authors declared no potential conflicts of interest with respect to the research, authorship, and/or publication of this article.

\section{Funding Statement}

The author received no financial support for the research and/or authorship of this article.

\section{Ethical Approval: Approved}

\section{REFERENCES}

1. World Health Organization. Pandemic fatigue reinvigorating the public to prevent COVID-19. 2020. https://apps.who.int/iris/bitstream/handle/10 665/335820/WHO-EURO-2020-116040906-55390-eng.pd

2. International Council of Nurses (2020). The Global Nursing shortage and Nurse Retention. 2020. https://www.icn.ch/sites/default/files/inlinefiles/ICN\%20Policy\%20Brief_Nurse\%20Sh ortage\%20and\%20Retention_0.pdf

3. Hu D, Kong Y, Li W, Han Q, Zhang X, Zhu LX, Wan SW, Liu Z, Shen Q, Yang J, He HG. Frontline nurses' burnout, anxiety, depression, and fear statuses and their associated factors during the COVID-19 outbreak in Wuhan, China: A large-scale cross-sectional study. EClinicalMedicine. 2020 Jul 1;24:100424. https://doi.org/10.1016/j.eclinm.2020.10042 4

4. Labrague LJ. Pandemic fatigue and clinical nurses' mental health, sleep quality and job contentment during the covid-19 pandemic: The mediating role of resilience. Journal of nursing management. 2021 Oct;29(7):19922001.
5. Cambridge Dictionary. Pandemic. 2022. https://dictionary.cambridge.org/us/dictionar y/english/pandemic

6. Cambridge Dictionary. 2022. Fatigue. https://dictionary.cambridge.org/us/dictionar y/english/fatigue? $\mathrm{q}=$ fatigue +

7. Badre D. How we can deal with 'pandemic fatigue': The first step is to understand that it's not just about exhaustion or tirednessor depleting a mental resource. Scientific American. 2021.

8. World Health Organization. State of the World's Nursing 2020. WHO, Geneva.[Google Scholar]. 2020 Apr.

9. Millard E. How to not let pandemic fatigue turn into pandemic burnout. Everyday Health. Available online at: https://www. everydayhealth. com/coronavirus/how-tonot-let-pandemic-fatigue-turn-intopandemic-burnout. 2021.

10. Cruickshank, S. The ills of pandemic fatigue. Johns Hopkins Bloomberg School of Public Health. 2021. https://publichealth.jhu.edu/2021/the-ills-ofpandemic-fatigue

11. Sperling D. Ethical dilemmas, perceived risk, and motivation among nurses during the COVID-19 pandemic. Nursing Ethics. $2021 \quad$ Feb;28(1):9-22. https://doi.org/10.1177/0969733020956376

12. Chachula KM. A comprehensive review of compassion fatigue in pre-licensure health students: antecedents, attributes, and consequences. Current Psychology. 2020 Oct 15:1-3. https://doi.org/10.1007/s12144020-01122-3

13. Despres C, Brunin Y, Berthier F, Pili-Floury $\mathrm{S}$, Besch G. Prone positioning combined with high-flow nasal or conventional oxygen therapy in severe Covid-19 patients. Critical Care. 2020 Dec;24(1):12.https://doi.org/10.1186/s13054-02002926-2

14. Stieg, C. . Could you get PTSD from your pandemic experience? The long-term mental health effects of coronavirus. CNBC. 2020. https://www.cnbc.com/2020/04/17/longterm-mental-health-ptsd-effects-of-covid19-pandemic-explained.html

15. Shahri SS, Ghashghaee A, Behzadifar M, Bragazzi NL, Behzadifar M, Mousavinejad N, Ghaemmohamadi MS, Ebadi F, Seyedin H. Depression among Iranian nurses: A systematic review and meta-analysis. Medical journal of the Islamic Republic of 
Iran.

2017;31:130.

http://doi.org/10.14196/mjiri.31.130

16. Buheji M, Buhaid N. Nursing human factor during COVID-19 pandemic. Int J Nurs Sci. 2020 Aug;10(1):12-24. http://doi.org/10.5923/j.nursing.20201001.0 2

17. Centers for Disease Control and Prevention. Interim Operational Considerations for Public Health Management of Healthcare Workers Exposed to or with Suspected or Confirmed COVID-19: non-US Healthcare Settings. 2021. https://www.cdc.gov/coronavirus/2019ncov/hcp/non-us-settings/public-healthmanagement-hcw-exposed.html

18. Bandyopadhyay S, Baticulon RE, Kadhum M, Alser M, Ojuka DK, Badereddin Y, Kamath A, Parepalli SA, Brown G, Iharchane S, Gandino S. Infection and mortality of healthcare workers worldwide from COVID-19: a systematic review. BMJ global health. 2020 Dec 1;5(12):e003097. http://dx.doi.org/10.1136/bmjgh-2020003097

19. Schwerdtle PN, Connell CJ, Lee S, Plummer V, Russo PL, Endacott R, Kuhn L. Nurse expertise: A critical resource in the COVID-19 pandemic response. Annals of Global Health. 2020;86(1). http://doi.org/10.5334/aogh.2898

20. Lippi G, Henry BM, Bovo C, SanchisGomar F. Health risks and potential remedies during prolonged lockdowns for coronavirus disease 2019 (COVID-19). Diagnosis. 2020 Jun 1;7(2):85-90. https://doi.org/10.1515/dx-2020-0041

21. Giorgi G, Lecca LI, Alessio F, Finstad GL, Bondanini G, Lulli LG, Arcangeli G, Mucci N. COVID-19-related mental health effects in the workplace: a narrative review. International journal of environmental research and public health. 2020 Jan;17(21):7857. https://doi.org/10.1371/journal.pone.025095 8

22. Stokes F, Iskander R. Human rights and bioethical considerations of global nurse migration. Journal of bioethical inquiry. 2021 Sep;18(3):429-39. https://doi.org/10.1007/s11673-021-10110-6

23. Mckeown B, Poerio GL, Strawson WH, Martinon LM, Riby LM, Jefferies E, Mccall C, Smallwood J. The impact of social isolation and changes in work patterns on ongoing thought during the first COVID-19 lockdown in the United Kingdom. Proceedings of the National Academy of Sciences. $2021 \quad$ Oct 5;118(40). https://doi.org/10.1073/pnas.2102565118

24. Bani-Issa WA, Al Nusair H, Altamimi A, Hatahet S, Deyab F, Fakhry R, Saqan R, Ahmad S, Almazem F. Self-Report Assessment of Nurses' Risk for Infection After Exposure to Patients With Coronavirus Disease (COVID-19) in the United Arab Emirates. Journal of Nursing Scholarship. $2021 \quad$ Mar;53(2):171-9. https://doi.org/10.1111/jnu.12625

25. O'Connor RC, Wetherall K, Cleare S, McClelland H, Melson AJ, Niedzwiedz CL, O'Carroll RE, O'Connor DB, Platt S, Scowcroft E, Watson B. Mental health and well-being during the COVID-19 pandemic: longitudinal analyses of adults in the UK COVID-19 Mental Health \& Wellbeing study. The British Journal of Psychiatry. $2021 \quad$ Jun;218(6):326-33. http://doi.org/10.1192/bjp.2020.212

26. Chirico F, Nucera G, Magnavita N. Protecting the mental health of healthcare workers during the COVID-19 emergency. BJPsych International. 2021 Feb;18(1). http://doi.org/10.1192/bji.2020.39

27. Chor WP, Ng WM, Cheng L, Situ W, Chong JW, Ng LY, Mok PL, Yau YW, Lin Z. Burnout amongst emergency healthcare workers during the COVID-19 pandemic: A multi-center study. The American Journal of Emergency Medicine. 2021 Aug;46:700. http://doi.org/10.1016/j.ajem.2020.10.040

28. Dobson H, Malpas CB, Burrell AJ, Gurvich C, Chen L, Kulkarni J, Winton-Brown T. Burnout and psychological distress amongst Australian healthcare workers during the COVID-19 pandemic. Australasian Psychiatry. $2021 \quad$ Feb;29(1):26-30. https://doi.org/10.1177/1039856220965045

29. Walker, L.O. \& Avant, K. C. Strategies for Theory Construction in Nursing (6th ed.). 2019. New York City, NY: Pearson.

30. Obeid, Z., \& Azar, N. A. Employeremployee relationships amid covid-19 pandemic. Lexology. 2020. https://www.lexology.com/commentary/em ployment-immigration/lebanon/obeid-lawfirm/employer-employee-relationshipsamid-covid-19-pandemic.

31. Marquedant, K. Study reveals the risk of COVID-19 infection among health care 
workers. Massachusetts General Hospital. 2020.

https://www.massgeneral.org/news/coronavi rus/study-reveals-risk-of-covid-19-

infection-among-health-care-workers.

32. Haddad, L. M., Annamaraju, P., \& ToneyButler, T. J. Nursing shortage. StatPearls. 2020.

https://www.ncbi.nlm.nih.gov/books/NBK4 93175/

33. Cullen, W., Gulati, G., \& Kelly, B. D.. Mental health in the COVID-19 pandemic. QJM: An International Journal of Medicine, 2020.113(5), 311-312. https://doi.org/10.1093/qjmed/hcaa110

34. Sampaio F, Sequeira C, Teixeira L. Nurses' mental health during the Covid-19 outbreak: a cross-sectional study. Journal of occupational and environmental medicine. $2020 \quad$ Oct 1;62(10):783-7. http://doi.org/10.1097/JOM.0000000000001 987

35. Ahorsu DK, Lin CY, Imani V, Saffari M, Griffiths MD, Pakpour AH. The fear of COVID-19 scale: development and initial validation. International journal of mental health and addiction. 2020 Mar 27:1-9. https://doi.org/10.1007/s11469-020-00270-8

36. Kameg BN. Psychiatric-mental health nursing leadership during coronavirus disease 2019 (COVID-19). Journal of Psychiatric and Mental Health Nursing. 2020 Jun 14. http://doi.org/10.1111/jpm.12662

37. Labrague LJ, De los Santos JA. COVID-19 anxiety among front-line nurses: Predictive role of organisational support, personal resilience and social support. Journal of nursing management. 2020 Oct;28(7):165361. https://doi.org/10.1111/jonm.13121

38. Pappa S, Ntella V, Giannakas T, Giannakoulis VG, Papoutsi E, Katsaounou P. Prevalence of depression, anxiety, and insomnia among healthcare workers during the COVID-19 pandemic: A systematic review and meta-analysis. Brain, behavior, and immunity. 2020 Aug 1;88:901-7. https://doi.org/10.1016/j.bbi.2020.05.026

39. Stamu-O'Brien C, Carniciu S, Halvorsen E, Jafferany M. Psychological aspects of COVID-19. Journal of Cosmetic Dermatology. 2020 Sep;19(9):2169-73. https://doi.org/10.1111/jocd.13601

40. Usher K, Durkin J, Bhullar N. The COVID-19 pandemic and mental health impacts. International journal of mental health nursing. 2020 Jun;29(3):315. http://doi.org/10.1111/inm.12726

41. Rogers JP, Chesney E, Oliver D, Pollak TA, McGuire P, Fusar-Poli P, Zandi MS, Lewis G, David AS. Psychiatric and neuropsychiatric presentations associated with severe coronavirus infections: a systematic review and meta-analysis with comparison to the COVID-19 pandemic. The Lancet Psychiatry. 2020 Jul 1;7(7):611$27 . \quad$ https://doi.org/10.1016/s22150366(20)30203-0

42. Dzinamarira $T$, Mhango $M$, Dzobo $M$, Ngara B, Chitungo I, Makanda P, Atwine J, Nkambule SJ, Musuka G. Risk factors for COVID-19 among healthcare workers. A protocol for a systematic review and metaanalysis. Plos one. 2021 May 4;16(5):e0250958. https://doi.org/10.1371/journal.pone.025095 8

43. Montgomery AP, Azuero A, Patrician PA. Psychometric properties of Copenhagen Burnout Inventory among nurses. Research in nursing \& health. 2021 Apr;44(2):308-18. https://doi.org/10.1002/nur.22114

44. Smets EM, Garssen B, Bonke BD, De Haes JC. The Multidimensional Fatigue Inventory (MFI) psychometric qualities of an instrument to assess fatigue. Journal of psychosomatic research. 1995 Apr 1;39(3):315-25.

https://doi.org/10.1016/00223999(94)00125-O

45. Varni JW, Burwinkle TM, Szer IS. The PedsQL Multidimensional Fatigue Scale in pediatric rheumatology: reliability and validity. The Journal of rheumatology. 2004 Dec 1;31(12):2494-500. https://doi.org/10.1002/cncr.10428

46. Varni J, Burwinkle T, Katz E. Meeske et al., Dickinson, P., 2002. The PedsQL TM in pediatric cancer: reliability and validity of the pediatric quality of life inventory TM generic core scales, multidimensional fatigue scale, and cancer module. Cancer.;94:2090-106.

47. Varni JW, Limbers CA, Bryant WP, Wilson DP. The PedsQL ${ }^{\mathrm{TM}}$ Multidimensional Fatigue Scale in type 1 diabetes: feasibility, reliability, and validity. Pediatric diabetes. 2009 Aug;10(5):321-8. http://doi.org/10.1111/j.13995448.2008.00482.x 
48. Chalder T, Berelowitz C. Pawlikowska T. Development of a fatigue scale. $\mathrm{J}$ Psychosomatic Res. 1993;37:147-54. https://doi.org/10.1016/00223999(93)90081-P

49. Jackson C. The Chalder fatigue scale (CFQ 11). Occupational medicine. 2015 Jan
1;65(1):86-.

https://doi.org/10.1093/occmed/kqu168

How to cite this article: Elvira Teng-Costa, Catherine M. Macatangay, Neil N. Balubar et.al. Pandemic fatigue: a concept analysis. Int $J$ Health Sci Res. 2022; 12(3): 79-92. DOI: https://doi.org/10.52403/ijhsr.20220312 